\title{
Novel simple sequence repeats (SSRs) detected by ND-FISH in heterochromatin of Drosophila melanogaster
}

\author{
Ángeles Cuadrado* and Nicolás Jouve
}

\begin{abstract}
Background: In recent years, substantial progress has been made in understanding the organization of sequences in heterochromatin regions containing single-copy genes and transposable elements. However, the sequence and organization of tandem repeat DNA sequences, which are by far the majority fraction of D. melanogaster heterochromatin, are little understood.

Results: This paper reports that the heterochromatin, as well as containing long tandem arrays of pentanucleotide satellites (AAGAG, AAGAC, AATAT, AATAC and AACAC), is also enriched in other simple sequence repeats (SSRs) such as A, AC, AG, AAG, ACT, GATA and GACA. Non-denaturing FISH (ND-FISH) showed these SSRs to localize to the chromocentre of polytene chromosomes, and was used to map them on mitotic chromosomes. Different distributions were detected ranging from single heterochromatic clusters to complex combinations on different chromosomes. ND-FISH performed on extended DNA fibres, along with Southern blotting, showed the complex organization of these heterochromatin sequences in long tracts, and revealed subclusters of SSRs (several kilobase in length) flanked by other DNA sequences. The chromosomal characterization of $C, A A C, A G G, A A T, C C G, A C G$, AGC, ATC and ACC provided further detailed information on the SSR content of D. melanogaster at the whole genome level.

Conclusion: These data clearly show the variation in the abundance of different SSR motifs and reveal their nonrandom distribution within and between chromosomes. The greater representation of certain SSRs in

D. melanogaster heterochromatin suggests that its complexity may be greater than previously thought.
\end{abstract}

\section{Background}

One of the most enigmatic aspects of genome organization in multicellular eukaryotes is the regionalization of chromosomes into euchromatin and heterochromatin domains. Heterochromatin, originally named "junk DNA" because no coding function could be found for it, is now considered essential for the epigenetic maintenance of centromeric function as well as for other cellular, developmental and evolutionary processes [1-3]. In most eukaryotes, the main components of heterochromatin are families of highly tandem repeated DNA or satellite DNA organized as multiple copies of a monomer sequence arranged in a head to tail pattern over megabase-long arrays. Running from a few base pairs to more than $1 \mathrm{~kb}$

\footnotetext{
*Correspondence: angeles.cuadrado@uah.es

Department of Cell Biology and Genetics, University of Alcalá de Henares, 28871 Alcalá de Henares, Madrid, Spain
}

in length, repeated units show a wide range of sizes and complexity. Despite sequence divergence between monomers of the same family often being very low over long arrays, satellite DNA in heterochromatin can change rapidly in nucleotide sequence or copy number during evolution. As a consequence, large numbers of unrelated satellite DNA families commonly compound the profile of satellite DNA in genomes $[4,5]$.

Drosophila melanogaster provides a model for studies of heterochromatin. About $59 \mathrm{Mb}$ of the $176 \mathrm{Mb}$ female genome, including the proximal half of the $\mathrm{X}$ chromosome, the pericentromeric region of autosomes 2 and 3, and most of the dot-like chromosome 4 is heterochromatic. The entire male $\mathrm{Y}$ chromosome $(41 \mathrm{Mb})$ is also heterochromatic [6]. However, polytene chromosomes, which have proved useful in mapping euchromatin regions, provide minimal resolution in heterochromatin 
analyses. Due to its late replication, the heterochromatin remains immersed in the diffuse and unbanded chromocentre region. Thus, mitotic rather than polytene chromosomes are preferable for chromosome mapping of heterochromatic sequences [7].

Over the last three decades, different families of highly repeated tandem sequences have been molecularly characterized and physically mapped to the chromosomes of D. melanogaster using different cloning strategies. Most of the highly repeated families of the fruit fly genome are composed of short repeat units 5-12 bp long, arrayed in tandem and extended over several megabases of DNA. These highly repeated sequences are localized in specific segments of the heterochromatin, which may contain different sets of satellite DNA [8-11].

The goal of large-scale genome projects is to convert the initial draft of euchromatic sequences into a complete telomere-to-telomere sequence for each chromosome. The Drosophila Heterochromatin Genome Project has made substantial progress in identifying contiguous sequences of the heterochromatin regions containing single-copy genes and dense clusters of transposable elements. However, the sequence and organization of the highly tandem repetitive DNA fraction - the vast majority of $D$. melanogaster heterochromatin - is little known [12]. The apparent absence of identified highly repeated sequences in certain heterochromatic regions and BACs covering the extensive heterochromatin gaps, suggests that unknown classes of highly repetitive DNAs must be present in this fraction of the D. melanogaster chromosomes. To determine what they are will require new technologies [13].

Short motifs of 1-6 bp repeated in tandem are classified as SSRs or microsatellites [14,15]. Although the genomes of higher organisms contain some long microsatellites (up to 500 nucleotides), in general these polymorphic loci are no longer than 100 nucleotides. However, in many species, SSRs may also be organized into long stretches of nearly 100 to several thousand tandem units mainly clustered in the heterochromatin, also referred to as satellite SSRs [16,17]. For example, in many vertebrate species, the heterochromatic sex chromosomes are rich in clusters of GATA and GACA repeats [18]. Long arrays of AAGAG and AATAT are found in the heterochromatin of fruit fly chromosomes [10]. Moreover, GGAAT and CATTT repeats have been found in the satellite regions of human chromosomes [19]. There is some evidence that the origin and evolution of satellite families, including those with relatively complex and longer repeated units, can be explained by the autoreplicative properties of different SSRs [20].

In the present study, a recently reported, simple and highly efficient non-denaturing FISH method (ND-FISH) was used to localize the classic satellites (AAGAG, AAGAC, AATAT and AATAC) and AACAC repeats
[21]. Heterochromatic regions enriched in SSRs were also sought, and the physical distribution of 16 different SSR types (mono-, di-, tri- and tetranucleotide repeats) studied in both polytene and mitotic chromosomes. An NDFISH analysis employing DNA fibres was also performed and the SSRs characterized by Southern hybridization.

\section{Methods}

\section{Chromosome preparation}

Mitotic and polytene chromosomes of $D$. melanogaster were obtained using third instar larvae of a wild type strain kept in our laboratory for about 20 years. The brains and salivary glands of individual larvae were dissected out in $45 \%$ acetic acid before squashing on a clean microscope slide with a drop of $45 \%$ acetic acid. After removing the cover slips by freezing, the slides were air dried.

DNA fibres were obtained from disrupted nuclei following a derivative of the alkaline/ethanol method described by Fidlerová et al. [22]. The brains of third instar larvae were dissected in a drop of water. Neuroblast tissues was disaggregated with a pestle in a drop of 3:1 ethanol:acetic acid on one end of a clean microscope slide. The resulting cell suspension was allowed to airdry. The slides were then rinsed in $100 \mathrm{ml}$ of phosphate-buffered saline solution (PBS) in a Coplin jar for 1 min. After removing the PBS solution (but not allowing the slide to dry out), the cell-containing area was incubated with $100 \mu \mathrm{l}$ of lysing buffer $(0.5 \mathrm{M} \mathrm{NaOH} /$ $30 \% \mathrm{ETOH}$ ) for $1 \mathrm{~min}$ to disrupt the nuclei before stretching the fibres by tilting the slide at $45^{\circ}$ and letting the buffer run away. $100 \mu \mathrm{l}$ of $100 \%$ ethanol were then added to the cell-containing area before post-fixation employing an ethanol series $(70 \%, 100 \%)$ (performed in a Coplin jar), allowing $5 \mathrm{~min}$ in each solution. Finally, the slides were air-dried.

\section{Probes and labelling}

Table 1 shows the twenty one oligonucleotide probes used. These were obtained from different companies, and had biotin and/or digoxigenin incorporated at their ends.

\section{Non-denaturing fluorescence in situ hybridization}

ND-FISH was performed as previously described [21]. Briefly, preparations were incubated at $24^{\circ} \mathrm{C}$ in a humidity chamber for $2 \mathrm{~h}$ with $30 \mu \mathrm{l}$ of hybridization buffer containing $2 \mathrm{pm}$ of the probe in $2 \times \mathrm{SSC}$. For post-washing, slides were immersed in $4 \times \mathrm{SSC} / 0.2 \%$ Tween $^{20}$ and agitated for $10 \mathrm{~min}$ at room temperature (RT). The detection of digoxigenin and biotin was respectively undertaken by incubating the slides in fluoresceinated anti-digoxigenin (Roche Applied Science) or streptavidin-Cy3 (Sigma) in $5 \%(w / v)$ BSA for $1 \mathrm{~h}$ at $37^{\circ} \mathrm{C}$. Before 
Table 1 Oligodeoxyribonucleotides used as probes to analyze SSRs in the present study and references of works where the distribution of SSRs has been reported previously by in situ hybridization in D. melanogaster chromosomes

\begin{tabular}{|c|c|c|c|}
\hline \multirow[t]{2}{*}{ SSRs } & \multirow[t]{2}{*}{ Probes used in study } & \multicolumn{2}{|c|}{ References } \\
\hline & & $\begin{array}{l}\text { Mitotic } \\
\text { chromosomes }\end{array}$ & $\begin{array}{l}\text { Polytene } \\
\text { chromosomes }\end{array}$ \\
\hline A & $\mathrm{A}_{20}$ & & $(21,40)$ \\
\hline C & $C_{20}$ & & $(40)$ \\
\hline$A C ; A G$ & $(A C)_{10} ;(A G)_{10}$ & (27) & $(27,40)$ \\
\hline $\begin{array}{l}\text { AAC; AAG; AAT, AAC; AGC; ACT; AGC; AGG; } \\
\text { ATC; CCG }\end{array}$ & $\begin{aligned}(\mathrm{AAC})_{5 ;} ;(\mathrm{AAG})_{5} ;(\mathrm{AAT})_{5 ;} & (\mathrm{ACC})_{5 ;}(\mathrm{ACG})_{5 ;}(\mathrm{ACT})_{5 ;}(\mathrm{AGC})_{5 ;}(\mathrm{AGG})_{5} ; \\
& (\mathrm{ATC})_{5 ;}(\mathrm{CCCG})_{5}\end{aligned}$ & & \\
\hline GACA & $(\mathrm{GACA})_{5}$ & & (21) \\
\hline GATA & $(\text { GATA })_{5}$ & (31) & (21) \\
\hline AAGAC; AAGAG; AATAC; AATAT & 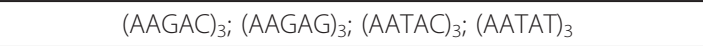 & (10) & \\
\hline AACAC & $(\mathrm{AACAC})_{3}$ & (11) & (11) \\
\hline
\end{tabular}

staining the DNA with DAPI, the slides were rinsed for 10 min in $4 \times \mathrm{SSC} / 0.2 \%$ Tween $^{20}$ at RT. The slides were then mounted in antifade solution (Vector Laboratories).

\section{Fluorescence microscopy and imaging}

Slides were examined using a Zeiss Axiophot epifluorescence microscope. For each motifs we analysed at least 10 cells. The images from the biotin/Cy3, digoxigenin/ Fitc and DAPI staining procedures were recorded separately using a cooled CCD camera (Nikon DS). The exposure times were variable depending of the intensity of the in situ signals obtained with each probe. The localization of the signals relative to the DAPI banding pattern was resolved by merging digital images using Adobe Photoshop. The length of single DNA fibres was measured taking into account that a beaded, interrupted signal often denotes a maximally decondensed DNA

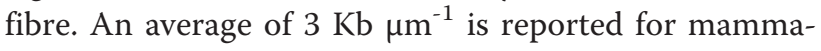
lian and Drosophila DNA [23,24].

\section{Southern blot analyses}

Genomic DNA was isolated from adult flies using the DNeasy Blood \& Tissue kit (Qiagen). DNA from both males and females $(7.5 \mu \mathrm{g})$ was digested with $A l u \mathrm{I}, R s a \mathrm{I}$ and Hinfl restriction endonucleases, divided into three aliquots and separated on 1\% agarose gel. After electrophoresis the gels were blotted onto nylon membranes (Biodyne, Pall Corporation) under alkaline conditions following standard techniques. Each membrane was cut into three and hybridized successively with different digoxigenin-labelled oligonucleotides as probes. To ensure high stringency, the temperature of hybridization and the post-hybridization washes were estimated for each oligonucleotide probe according to the $\mathrm{Tm}$ and adhering to the $\mathrm{GC}$ content rule: $30.7^{\circ} \mathrm{C}$ for $\mathrm{AATAC}_{3}$, $36.2^{\circ} \mathrm{C}$ for $\mathrm{ACT}_{5}, \mathrm{AAG}_{5}$ and $\mathrm{AAC}_{5}, 38.9^{\circ} \mathrm{C}$ for $\mathrm{AAGAG}_{3}, \mathrm{AACAC}_{3}$ and $\mathrm{AAGAC}_{3}, 44^{\circ} \mathrm{C}$ for $\mathrm{GATA}_{5}$, and $54.3^{\circ} \mathrm{C}$ for $\mathrm{AG}_{10}, \mathrm{AC}_{10}$ and $\mathrm{GACA}_{5}$. Hybridization was performed overnight using $2 \mathrm{pm}$ of oligonucleotides in $5 \times \mathrm{SSC}, 0.02 \% \mathrm{SDS}, 0.01 \% \mathrm{LSS}$ and $0.3 \%$ blocking reagent (Roche). Filters were washed with $0.15 \times$ SSC and $0.1 \%$ SDS at the corresponding Tm for $30 \mathrm{~min}$ before detection using anti-digoxigenin-AP, Fab fragments (Roche), and CDP-Star (Roche) according to the manufacturer's recommendations. The techniques followed were basically as described in Loarce et al. [25].

\section{Results}

Chromosomal localization of pentanucleotide DNA satellites by ND-FISH

To verify whether ND-FISH was a suitable technique for identifying clusters of SSRs in the heterochromatin of D. melanogaster, the chromosomal distributions of five pentanucleotide probes were analyzed: (AAGAG) 3 , $(\mathrm{AAGAC})_{3},(\mathrm{AATAT})_{3},(\mathrm{AATAC})_{3}$ and $(\mathrm{AACAC})_{3}$ (Figure 1). These probes were chosen because they share up to $80 \%$ homology and represent well characterized families of satellite DNA, which differ in abundance, complexity and chromosomal distribution. The neuroblasts and salivary gland cells obtained from individual larvae (squashed on the same slide) were analysed. This allowed the distribution and intensity of the in situ signals obtained simultaneously with each probe in both diploid and polytene nuclei to be compared (Figure 1a and $1 \mathrm{~m}$ ).

The AAGAG probe was found at different intensities in multiple clusters in the chromocentre of polytene chromosomes and in interphase neuroblast nuclei (Figure 1a-c and 1e). All mitotic chromosomes showed in situ signals (Figure $1 \mathrm{~b}$ and $1 \mathrm{e}$ ). The number of clusters on chromosome 2 could not be determined due to the proximity of their signals, which were arranged in a dispersed pattern along most of the heterochromatin (het) of the right arm (2Rhet). The multiple sites observed on the Y chromosome were concentrated in three regions along its length, with two on $\mathrm{YL}$ and one on $\mathrm{Y}^{\mathrm{S}}$. AAGAG repeats were also 

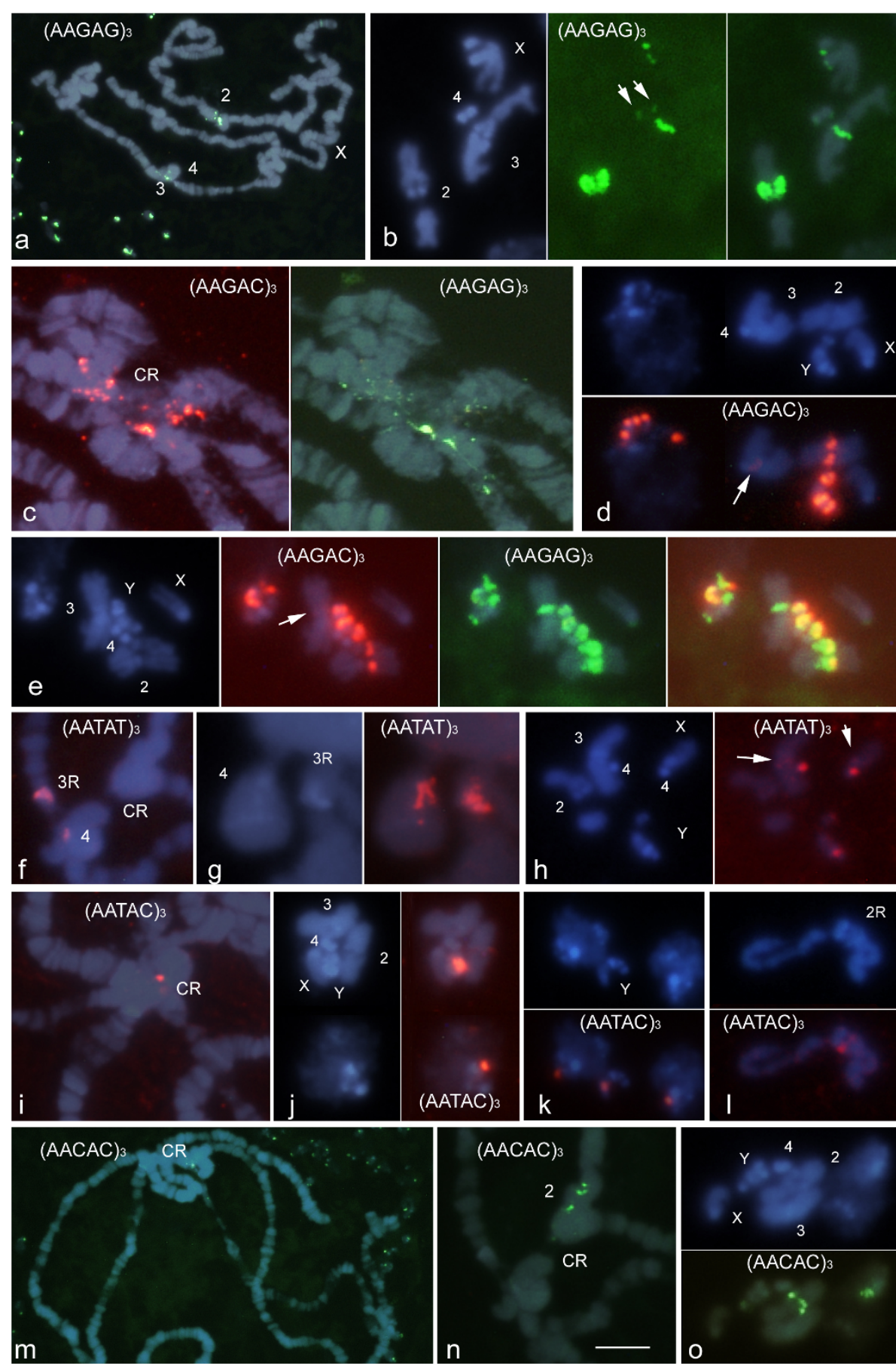

Figure 1 Photomicrographs showing the motif-dependent chromosomal distribution of the pentanucleotide probes in the salivary gland ( $a, c, f, g, i, m$ and $n$ ) and neuroblast cells ( $a, b, d, e, h, j, k, l, m$ and o) of male and female D. melanogaster after ND-FISH and DAPI staining. Each panel shows individual and/or merged images to facilitate the visualization of the signals (green or red for digoxigeninand biotin-labelled probes respectively) with respect to the DAPI (blue) banding pattern. In some samples interphase and mitotic neuroblast nuclei are shown in the same panels; both polytene and diploid nuclei are shown in a and $\mathbf{m}$. Mitotic chromosomes are identified as well as polytene regions of interest. An example of two-colour ND-FISH is shown in e. Note that only the diffuse chromocentre (CR) observed in polytene nuclei is enriched in pentanucleotide SSRs, which localize to specific heterochromatic regions in mitotic chromosomes as shown in Figure 4. The arrows point to low intensity signals. Scale bar: $5 \mu \mathrm{m}$, except in $\mathbf{a}$ and $\mathbf{m}$, in which it represents $25 \mu \mathrm{m}$. 
found in significant amounts on 3Rhet and in two minor clusters, one distal on XR and the other on chromosome 4.

$(\mathrm{AAGAC})_{3}$ showed a similar chromosomal distribution to (AAGAG) 3 . However, when two-colour ND-FISH was performed using both probes simultaneously, differences were observed between the polytene and mitotic chromosomes (Figure 1c and 1e). In male metaphases, as with the AAGAG probe, multiple sites were observed on the $\mathrm{Y}$ chromosome, concentrated in the same regions (Figure 1e). The AAGAC repeats were localized in a strong cluster on 2 Rhet and in a minor site on 3 Rhet (Figure 1d).

The AATAT probe was also found in the chromocentre of polytene chromosomes (Figure 1f). A detailed analysis allowed the AATAT repeats to be localized precisely at the base of the polytenized regions of chromosomes 3R and 4 (Figure 1g). In mitotic chromosomes these repeats were primarily found on chromosome 4 . In addition, multiple sites of lower intensity were detected on chromosome $\mathrm{Y}$ (the strongest at the two tips). Two minor clusters were also seen, one distal on $\mathrm{XR}$ and the other on 3R, presumably near the euchromatin-heterochromatin junction (Figure $1 \mathrm{~h}$ ).

The AATAC probe was detected in the chromocentre of polytene chromosomes (Figure 1i). In mitotic chromosomes, a strong cluster on YL (Figure $1 \mathrm{j}-\mathrm{k}$ ) and a minor site on 2Rhet were observed (Figure 11).

Finally, a few AACAC signals were found dispersed in the chromocentre (Figure $1 \mathrm{~m}$ ), in the unbanded regions of chromosome arm $2 \mathrm{R}$ (Figure $1 \mathrm{n}$ ). In metaphase chromosomes these repeats were situated on 2 Rhet and $Y^{S}$ (Figure 10).

\section{Chromosomal localization of mono- and dinucleotide SSRs by ND-FISH}

Figure 2 shows the distribution of the homonucleotide probes $A_{20}$ and $C_{20}$, which detect arrays of $A / T$ and $\mathrm{C} / \mathrm{G}$ mononucleotides respectively as well as two alternating nucleotide repeats: $(\mathrm{AG})_{10}$ and $(\mathrm{AC})_{10}$. The analysis of the other two possible dinucleotide repeats, AT and CG, could not be performed accurately using hybridization techniques owing to their self-complementing.

The $A_{20}$ probe showed a great number of signals in polytene chromosomes. A high density of A repeats was seen on the $\mathrm{X}$ chromosome, especially concentrated near the chromocentre (Figure 2a), as previously reported (Table 1, [21]). In mitotic chromosomes, $\mathrm{A}_{20}$ was found interstitially along chromosome arm XL and in three clusters on chromosome $\mathrm{Y}$, with two on the long arm and one in its satellite (Figure 2c). A disperse distribution of A signals was observed throughout the euchromatin in mitotic chromosomes when using long image capturing exposure times. The highest concentration was seen on the distal half of chromosome X, contrasting with the absence of signals on chromosome 4. In addition, higher concentrations of dispersed signals were observed in the proximal chromosome arm $2 \mathrm{R}$ (Figure 2b).

Polytene chromosomes hybridized weakly with the $\mathrm{C}$ probe. However, with the help of the CCD camera, these repeats were observed as many bands that decreased in number towards the chromocentre, and which were particularly concentrated on the X chromosome (Figure 2d).

With the exception of chromosome 4, a large number of intense signals for $(\mathrm{AC})_{10}$ were seen along the length of the polytene chromosomes arms. As described above for the mononucleotide repeats, the density of signal along the $\mathrm{X}$ chromosome was higher. In addition, a few signals of different intensity were found dispersed in the chromocentre, some in the unbanded regions of chromosome 2 (Figure 2e). In mitotic chromosomes, weak signals were seen dispersed over most of the euchromatin. A cluster of stronger signals was seen near the centromere of chromosome arm 2R (Figure 2f).

The distribution and intensity of the (AG) 10 signals fit the general pattern described for AC repeats in polytene chromosomes (Figure 2g-h). In addition, the AG probe was found in multiple clusters of different intensity in the chromocentre. The unbanded region of chromosome 2 was particularly rich (Figure $2 \mathrm{~g}$-h). AG repeats were detected in multiple clusters in all mitotic chromosomes. Several sites were observed on chromosome 2, especially concentrated on 2 Rhet (Figure $2 \mathrm{j}$ ), and in the two tips of chromosome Y. A cluster was also found on 3Rhet along with two minor sites on chromosome 4 and another on the tip of chromosome XR (Figure 2i).

\section{Chromosomal localization of tri- and tetranucleotide SSRs by ND-FISH}

Probes with all the possible trinucleotidic combinations were analysed by ND-FISH. Well-defined signals were observed only for $(\mathrm{AAG})_{5},(\mathrm{ACT})_{5}$ and $(\mathrm{AAT})_{5}$, which appeared restricted to a few unambiguous sites on specific arms of polytene chromosomes (Figure $3 \mathrm{a}, \mathrm{d}$ and $3 \mathrm{~g}$ respectively). The AAG and ACT repeats gave signals clustered in the chromocentre (Figure $3 \mathrm{a}$ and $3 \mathrm{~d}$ respectively). In mitotic chromosomes, (AAG) $)_{5}$ hybridized at three sites on chromosome arm 2Rhet, two on YL and one on $Y^{S}$. In addition, a less intense site was observed on 3Rhet and two small sites were seen on chromosomes 4 and X (Figure $3 \mathrm{~b}-\mathrm{c}$ ). ACT repeats were exclusively found on 2Rhet (Figure 3f). The most intense AAT signals were seen in the interstitial regions of chromosome $3 \mathrm{R}$ and on chromosome 4, and near the chromocentre of chromosome $\mathrm{X}$ in polytene chromosomes (Figure $3 \mathrm{~g}$ ). A detailed analysis using high quality chromosome spreads showed clusters near the base of chromosome X (Figure 3h). 


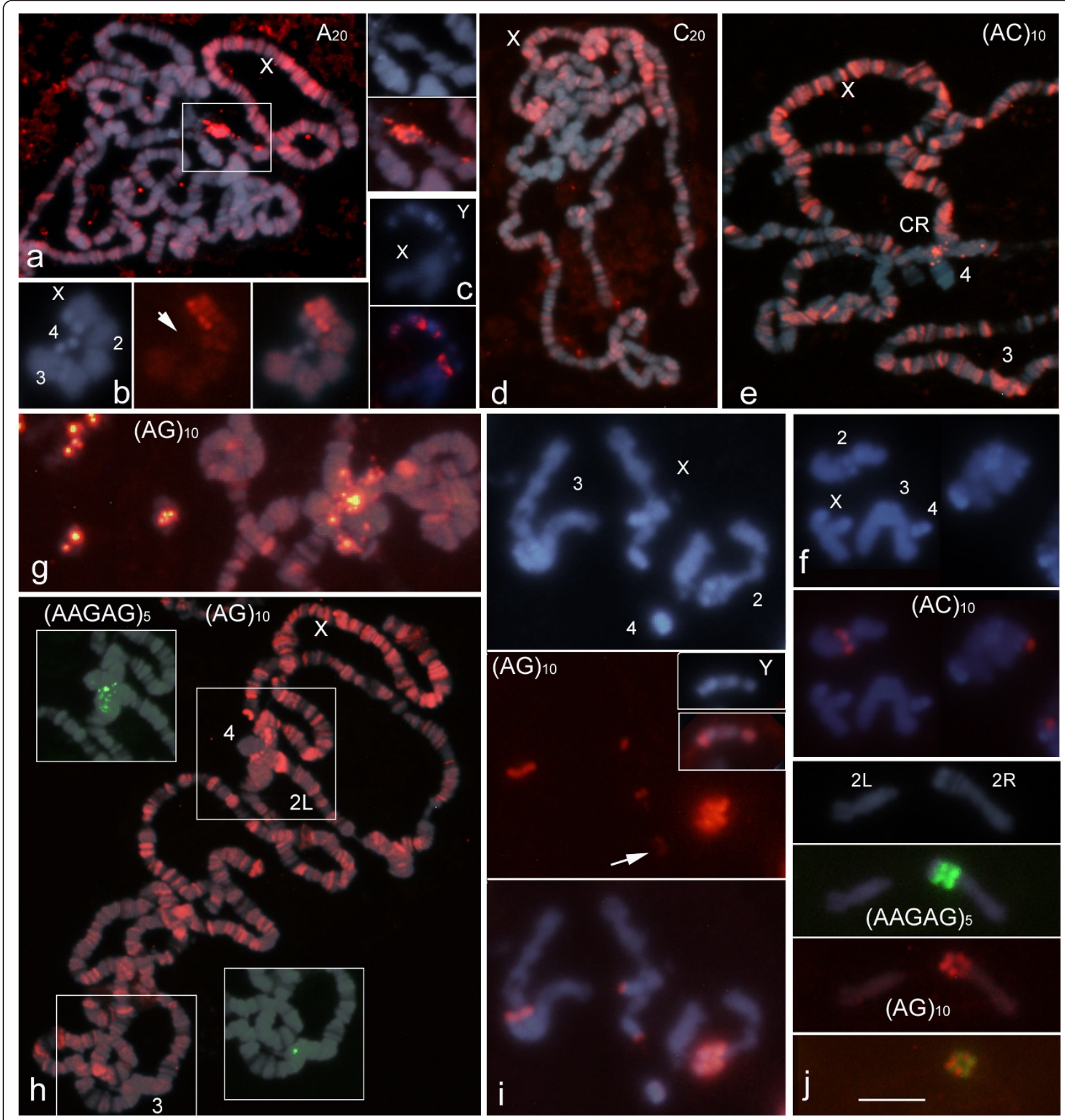

Figure 2 Chromosomal distribution of the mono- and dinucleotide SSRs in the salivary gland (a, d, e, and h) and neuroblast cells (b, c, $\mathbf{f}, \mathbf{i}$ and $\mathbf{j}$ ) of $D$. melanogaster after ND-FISH and DAPI staining. Both polytene chromosomes and diploid nuclei are shown in $\mathbf{g}$; examples of two-colour ND-FISH with (AAGAG) $)_{5}$ are shown in $\mathbf{h}$ (insets) and $\mathbf{j}$. Each panel shows individual and/or merged images to facilitate the appreciation of the distribution of signals (green or red from digoxigenin- and biotin-labelled probes respectively) and identification of chromosomes. The localization of the signals with respect to the heterochromatic DAPI banding pattern in mitotic chromosomes is shown in Figure 4. Note the high concentration of A, C, AC and AG SSRs on polytene X chromosomes; this contrasts with the lesser presence of these repeats on chromosome 4 . An enlarged view of the $A$ repeat signals at the base of chromosome $X$ (square) and chromosome 4 is shown at the right of panel a. The arrows point chromosomes 4; CR, chromocentre. Scale bar $=5 \mu \mathrm{m}$ and $25 \mu \mathrm{m}$ in (pro)metaphase and polytene nuclei respectively. 


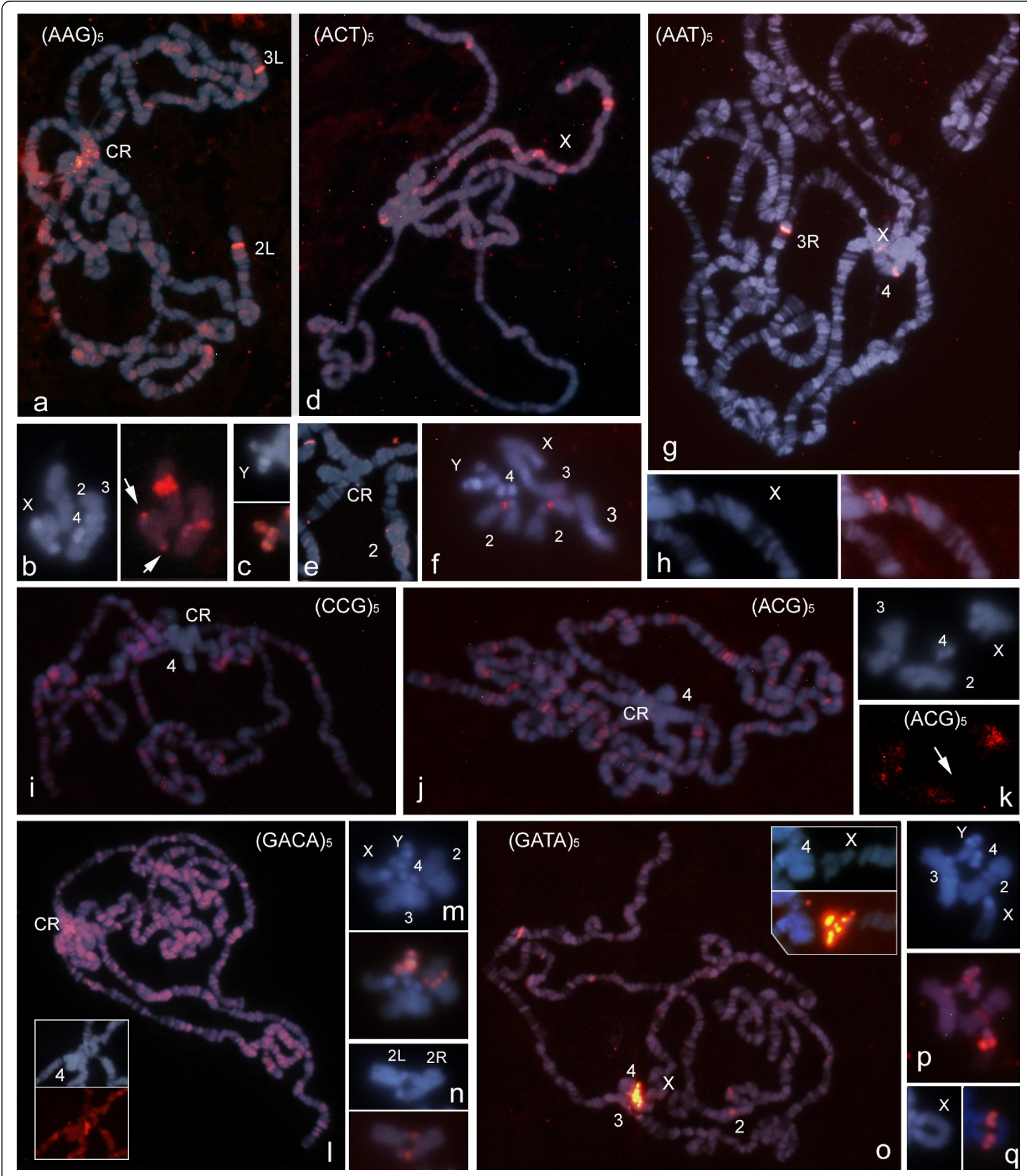

Figure 3 The non-random chromosomal distribution of several tri- and tetranucleotide SSRs in polytene (panels a, d, e, g, h, i, j, I and o; scale bar $25 \mu \mathrm{m}$ ) and mitotic (b, c, f, k, m, n, p and q; scale bar $5 \mu \mathrm{m}$ ) nuclei of $D$. melanogaster, as shown by ND-FISH with the indicated biotinylated probes. Each panel shows individual and/or merged images to facilitate the visualization of the red signals with respect to the DAPI (blue) staining and to allow chromosome identification. The view of the localization of the AAT repeats in the base of chromosome $X$ is enlarged in $\mathbf{h}$. A detailed view of the chromocentre (CR) after ND-FISH with (ACT) $)_{5}(G A C A)_{4}$ and $(G A T A)_{4}$ is shown in $\mathbf{e}$ and in the inserts in panels $\mathbf{I}$ and $\mathbf{o}$ respectively. The arrows point to chromosomes $X$ and 4 in $\mathbf{b}$ and $\mathbf{k}$ respectively. 
Increasing the $\mathrm{CCD}$ camera exposure time revealed specific patterns of low intensity signals for AAC, ACC, ATC, ACG, AGC, AGG and CCG distributed as a large number of signals along the length of the polytene arms (Figure 3i and j). Regardless of differences in the abundance and localization of each motif on the polytene chromosomes, a uniform pattern of distribution was observed over all chromosome arms, but particularly on chromosome $\mathrm{X}$. The use of very long exposure times revealed a characteristic dispersion pattern of signals in mitotic chromosomes (Figure 3k).

ND-FISH was used in previous work to determine the distribution of (GACA $)_{5}$ and (GATA $)_{5}$ in polytene chromosomes of D. melanogaster ([21] and Figure 31 and 30 respectively). That analysis was here extended by analysing mitotic chromosomes (Figure $3 \mathrm{~m}-\mathrm{n}$ and $3 \mathrm{p}-\mathrm{q}$ respectively). In addition to the high concentration of GACA repeats found in the chromocentre of polytene chromosomes, a rich pattern of signals of similar intensity was seen over the chromosome arms (Figure 3l). The strongest (GATA) signals were detected at the chromocentre and a few specific sites on the chromosomes arms (Figure 3o). GACA repeats were especially numerous on mitotic chromosomes 2Rhet and Y (two clusters on YL and one site on its satellite). A minor cluster was also detected on chromosome 3Rhet. The strongest GATA signals were seen on the long arm of chromosome $\mathrm{X}$, with minor sites on chromosomes 2, 4 and Y (Figure 3p-q).

\section{Location of SSRs relative to cytogenetic heterochromatic regions}

Figure 4 shows the chromosomal distribution of all the SSRs in the heterochromatin of D. melanogaster using a map adapted from Pimpinelli et al. [26] which recognizes 61 cytogenetic regions by DAPI banding. The localization of the AAGAG, AAGAC, AATAT and AATAC repeats agrees with that shown in the map of Lohe et al. [10], while the distribution of the AACAC repeats agrees with the map produced by Makuni et al. [11] Although several clusters of different repeat motifs were mapped to the same cytogenetic regions, the last two groups of authors were able to assign SSRs to segments of the heterochromatin using identified chromosome rearrangements and breaks in both the euchromatin and heterochromatin. With the exception of two additional clusters of AAGAC on 3Rhet and AATAC on 2Rhet, the localization of the pentanucleotide probes here analysed by ND-FISH are in good agreement with the results of the above authors, both in terms of appearance and chromosomal position (compare Figure 1 and 4). Two-colour ND-FISH clearly resolved the proximal-distal order of two probes with respect to the position of fluorescence signals within the cytogenetic map (Figure 4). For example, the previously unmapped clusters of AAGAC repeats found on 3Rhet co-localized to a proximal position with respect to a cluster of AAGAG, precisely mapped on segment h57 (Figure 1e). In the same way, the AATAC cluster detected on 2 Rhet was proximal to AACAC mapped in h42, presumably in region h41. This strategy was used to assign the remaining SSRs to heterochromatin segments.

The detailed analysis of polytene chromosomes allowed the SSRs clustered at the boundaries of the pericentromeric heterochromatin to be assigned to different heterochromatin segments. For example the AATAT repeats found near the chromocentre on $3 \mathrm{R}$ might correspond to the signal found on $3 R$ het near the frontier between the euchromatin and heterochromatin, presumably at the boundary of region h58 (Figure 1f). In the same way, the GATA repeats found near the chromocentre at the base of chromosome $\mathrm{X}$ might correspond to the signals clustered on the long arm of chromosome $\mathrm{X}$ at the heterochromatin-euchromatin border in the region h26 (Figures 3o).

\section{Analysis of DNA fibres by ND-FISH}

To better estimate the size and molecular organization of the novel SSR clusters in the heterochromatin, the extended chromatin fibres were studied. In initial experiments, the lysed nuclei of neuroblasts were used to examine the quality of the fibres (in this case not extended) and the possibility of using ND-FISH (Figure 5a). The hybridization signals were clear for the intact nuclei still condensed and the expanded DNA around the lysed nuclei. The resolution achieved for the disrupted nuclei was sufficient to co-localize different probes as non-intermingled adjacent blocks, even when they hybridized at the same chromosome positions. Further, when extended chromatin fibres from lysed nuclei were generated, ND-FISH revealed long arrays of fluorescent signals for different probes (Figure $5 \mathrm{~b}$ to $5 \mathrm{f}$ ). Condensed chromatin fibres appeared as thick continuous lines; thin fibres, observed as beaded, interrupted signals, are the result of maximally decondensed DNA fibres (Figure 5e). With both the classic pentanucleotide satellites and the other SSRs found clustered in heterochromatin, fibres with comparable lengths and similar beaded patterns were observed. Assuming an average of $3 \mathrm{~kb} \mathrm{\mu m}^{-1}$ for the extension of maximally stretched fibres, the largest fibres observed (extended over the field of view of the $100 \times$ objective) contained several hundred kilobases. These fibres commonly presented gaps of thousands of base pairs without hybridization flanked by regions showing fluorescent signals (Figure 5f).

\section{Molecular organization of SSRs in D. melanogaster}

The molecular organization of the SSR clusters was studied by Southern hybridization. Tests were performed using DNA probes labelled with digoxigenin and genomic DNA from male and female adult flies digested 

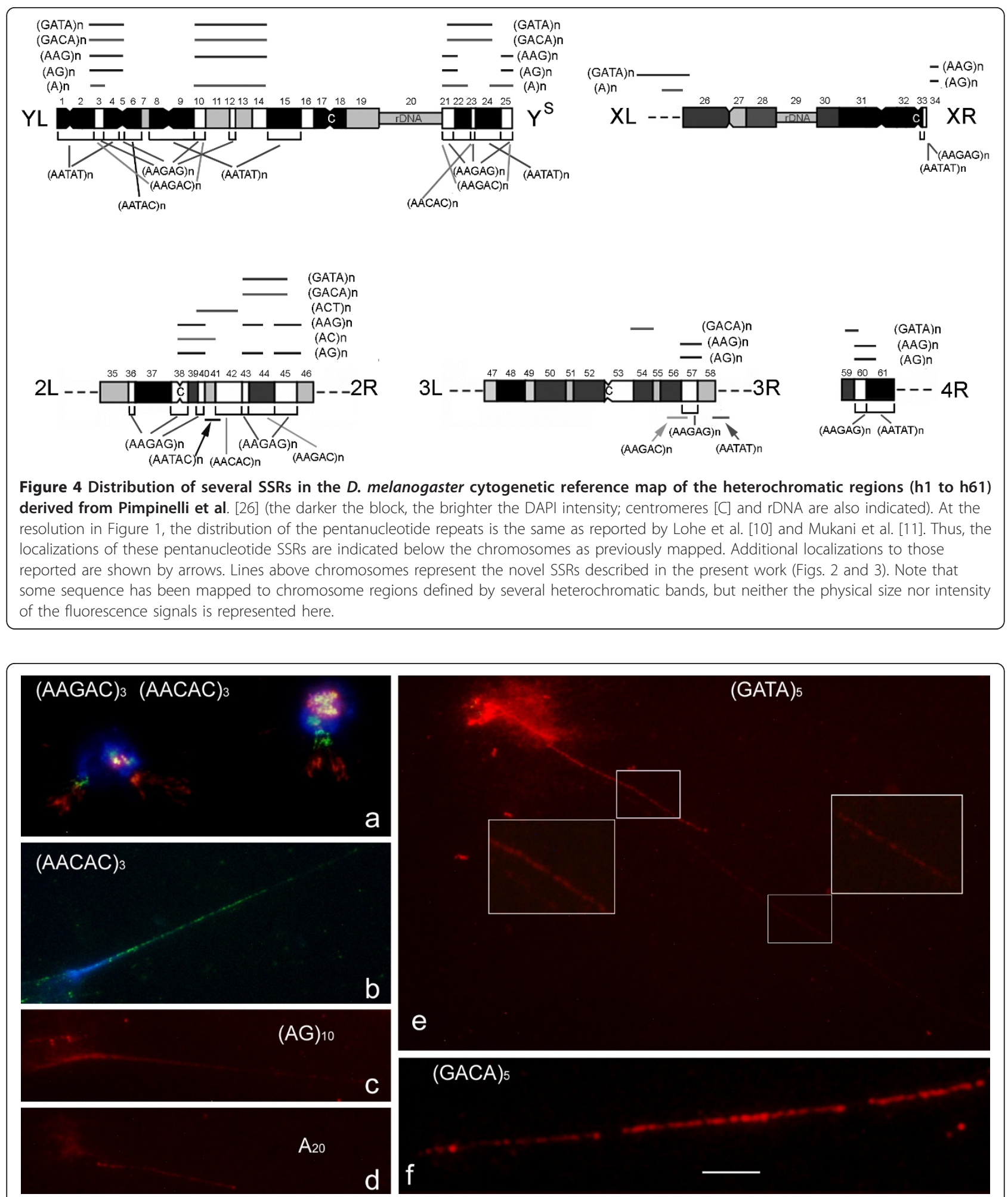

Figure 5 Organization of representative SSRs in extended DNA fibres from neuroblast nuclei of $D$. melanogaster after ND-FISH with the indicated probes (green or red signals from digoxigenin- and biotin-labelled probes respectively) and DAPI (blue) staining. An example of two colour fibre ND-FISH in haloed nuclei with $(\mathrm{AAGAC})_{3}$ (red) and (AACAC) ${ }_{3}$ (green) is shown in $\mathbf{a}$. Examples of fluorescent signal patterns in fibres stretched to different degrees are shown in e (square insets). Note the characteristic beaded nature of the signals in the highly extended fibres. A representative of the gaps observed between the continuous track of beaded signals is amplified in $\mathbf{f}$. Scale bar $=10 \mu \mathrm{m}$, except in $\mathbf{f}$ in which it represents $5 \mu \mathrm{m}$. Every micrometer represents $3 \mathrm{~kb}$ of the highly stretched DNA. 
separately with the restriction enzymes AluI, Hinfl and RsaI (Figure 6). The three restriction enzymes have specific recognition sites of four base pairs and digested most of the genomic DNA into fragments shorter than $1 \mathrm{~kb}$. DNA resistant to the endonucleases was observed at the exclusion limit in gel electrophoresis. Different patterns of fragments were revealed for the male and female genomic DNA (Panel A). The three endonucleases used had no recognition sites within the repeat sequences analysed, allowing the identification of the full SSR tracks and short flanking sequences.

Initially our Southern results show the abundance and size of the fragments to vary significantly between different SSRs. Only the pentanucleotide probes hybridized to the restriction enzyme-resistant DNA (Figure 6B-E), indicating the absence of the restriction sites and the existence of large arrays. Three noticeable differences were detected among the probes. (AAGAG) ${ }_{3}$ gave the strongest hybridization pattern with the genomic DNA of both sexes, with most of the fragments longer than $20 \mathrm{~kb}$ (Figure 6B), while a prominent male-specific hybridization pattern was observed with the (AAGAC) ${ }_{3}$ and (AATAC) $)_{3}$ probes (Figure 6C-D), defining the existence of the $\mathrm{Y}$ chromosome. Finally (AACAC) ${ }_{3}$ revealed multiple and well defined bands of hybridization ranging in size from 1 to $20 \mathrm{~kb}$.

The remaining SSR probes returned specific patterns of hybridization varying in number and fragment size.

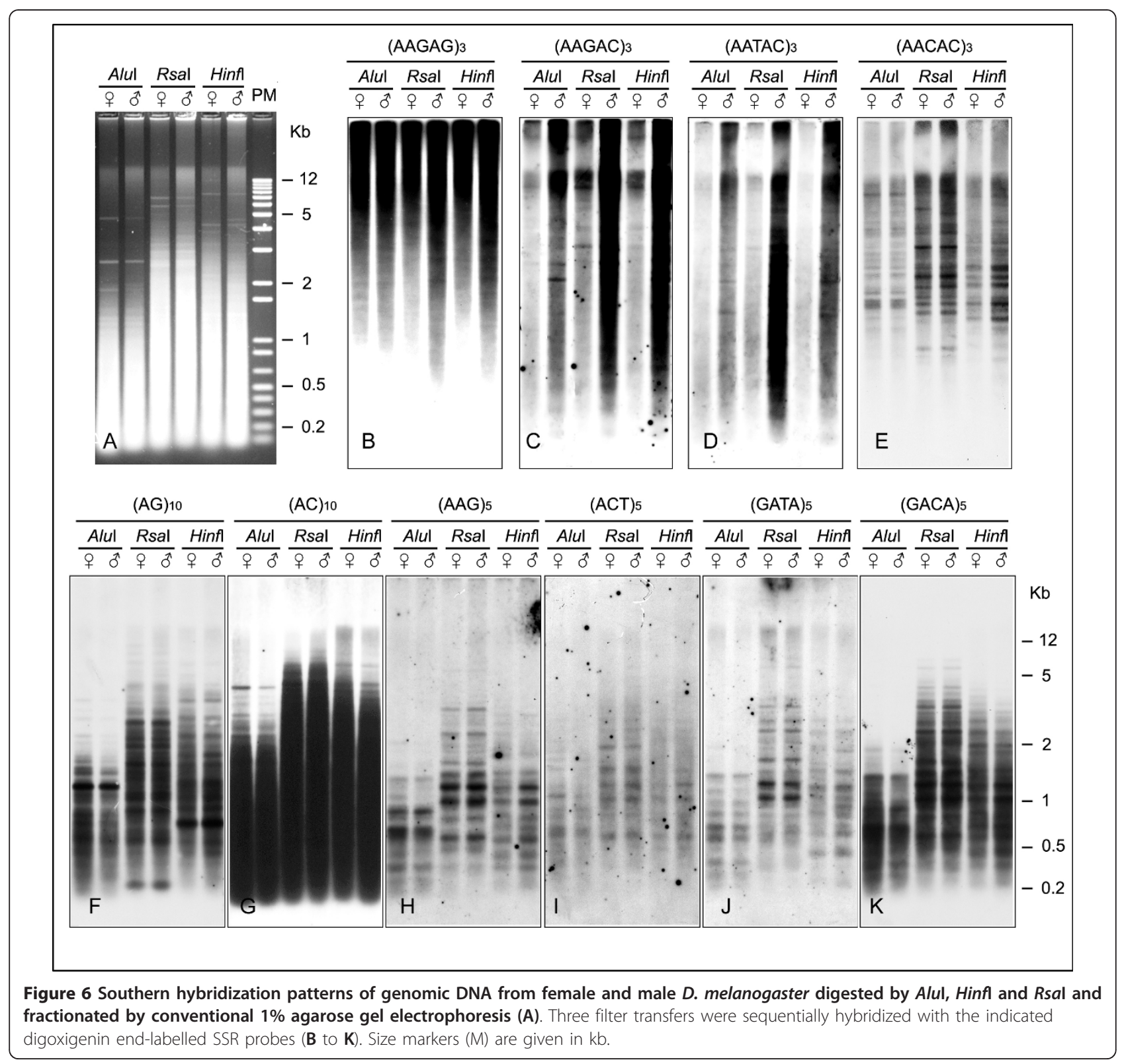


The $(\mathrm{AC})_{10}$ probe showed the strongest hybridization, with fragments ranging from a few base pairs to $5 \mathrm{~kb}$ in size (Figure 6F). $(\mathrm{AG})_{10},(\mathrm{AAG})_{5},(\mathrm{AAC})_{5},(\mathrm{ACT})_{5}$ $(\text { GATA })_{5}$ and $(\text { GACA })_{5}$ showed specific patterns with wide spectrums of well-defined fragments ranging from 0.1 to $4 \mathrm{~kb}$ in size (Figure $6 \mathrm{G}-\mathrm{K}$ respectively).

The remarkable differences between the motifs and the composite SSRs, for example AG and AAG and the composite AAGAG or AC and AG and the composite GACA, revealed by southern pattern (Figure 6), provide evidence for the specific amplification of each SSRs at particular chromosomal locations.

\section{Discussion}

Reliability of the ND-FISH technique in the visualisation of SSR-enriched chromosome regions

The five pentanucleotides SSRs used to analyze the classic DNA satellites of D. melanogaster differ from one another by at least one nucleotide. Therefore, it is to be expected that these probes might hybridize with one another under low stringency hybridization conditions. The contrasting distribution results for these SSRs show, however, that even probes with $80 \%$ identity provide motif-specific hybridization patterns in ND-FISH. Moreover, except for two faint additional signals with $(\mathrm{AATAC})_{3}$ and $(\mathrm{AAGAC})_{3}$, the results obtained here were in complete agreement with previous localizations involving clones containing the satellite repeats as probes in high stringency in situ hybridization experiments using other flies stocks ([10,11] and Figures 1 and 4$)$. These results suggest that heterochromatic SSRs clusters are conserved among strains. However we can no discard certain level of polymorphism (extents/distributions of SSRs) among different flies stocks. Additional clusters of AG and AC on mitotic chromosomes were detected beyond those discovered in previous work involving FISH and long duplex probes composed of simple motifs [27]. This result indicates that ND-FISH is a higher efficient technique improving the sensitivity of detection of SSRs enriched chromosome regions.

No attempt was made to construct a higher resolution physical map of SSR clusters in the heterochromatin. Even when analysing the heterochromatic regions in well spread prometaphase cells, it was impossible to reliably assign probes to specific blocks in DAPI-stained chromosomes (since there are about $100 \mathrm{Mb}$ of heterochromatin divided into 61 bands) (e.g., see Figure 1b and 2i). Further, due to the fluorescence spreading, it could not be determined whether strong signals were actually produced by two or more neighbouring SSR clusters. Nevertheless, Figure 4 provides a useful working map of the highly repetitive components of the D. melanogaster genome, which further analyses ought to improve (e.g., using simultaneous and/or sequential ND-FISH with different probes and analysing ordered chromosome rearrangements or chromosomes with deletions of specific heterochromatic bands, etc.).

The ND-FISH technique is simpler, faster and more efficient than any previously reported in situ method for analyzing the chromosome distribution of SSRs [21]. Another advantage of ND-FISH over standard FISH is that it avoids chromosome denaturation; thus, the morphology of the chromosomes is well maintained. This is very important when successive reprobing is required on the same slide. Moreover, ND-FISH probably prevents DNA losses. This is desirable for higher resolution mapping using DNA fibres, which often become fragmented during the denaturing step of standard FISH. Thus, ND-FISH resolves one of the main limitations of the analysis of DNA fibres by conventional FISH. The results in Figure 5 show that neuroblasts are an excellent source of nuclei for lysing, and that oligonucleotides used as probes in ND-FISH permit the mapping of SSRs in extended chromatin fibres.

\section{D. melanogaster heterochromatin is enriched in SSRs}

D. melanogaster is a model for heterochromatin studies and the euchromatin-heterochromatin boundaries have been fully analysed. Moreover, heterochromatic sequences of about $20 \mathrm{Mb}$, mainly containing singlecopy sequences and transposable elements, have been almost completely mapped $[13,28]$. However, as in other "finished" genomes, the sequence and structure of the main components of the heterochromatin, which contains highly repetitive tandem sequences, are poorly characterized [29].

As far as we know, the only SSRs molecularly characterized with a unit length under $4 \mathrm{bp}$ in the heterochromatin of $D$. melanogaster are TAA and GATA. TAA is involved in a complex sequence of $5.9 \mathrm{~kb}$ which maps to $\mathrm{h} 26$, the most distal division of the cytogenetic map [30]. The present data show a distribution of AAT very close to the proximal region of chromosome $\mathrm{X}$ (Figure $3 h$ ), suggesting it might be the same sequence array.

Singh et al. [31] reported the existence of GATA repeats concentrated in the proximal region of the $\mathrm{X}$ chromosome as a minor repeat class in the genome of D. melanogaster. The sequencing of chromosome $\mathrm{X}$ showed GATA to also be present in the pericentromeric heterochromatin ends while the most proximal sequence scaffold begin also in TAGA repeats, flanking a gap of unknown size in the heterochromatic region h26 [13]. The present ND-FISH data suggest that the stronger signals found with the (GATA) 5 probe at the euchromatin-heterochromatin boundary of the $\mathrm{X}$ chromosome (Figure 3o-q) might represent this unsequenced gap. 


\section{Structure of SSRs clusters}

Satellite DNA can be extended over megabases of DNA but the maximum length is unknown due to the lack of large DNA clones containing highly repeated sequences. For example, two large blocks of AATAT and AAGAG satellites ranging up to approximately $380 \mathrm{~kb}$, interrupted by five transposons, have been identified in the functional centromeric region of the minichromosome Dp1187 in D. melanogaster, but only contigs of about $8 \mathrm{~kb}$ of these repeats have been obtained [32,33].

Several kilobases of target sequence are required to produce consistent hybridization signals in metaphase chromosomes, although this does not mean that the target must exist in long interrupted stretches. On the other hand, no important differences were found between the probes here analysed for visualising the arrays of SSRs using DNA fibres (Figure 5). The characteristic appearance of a beaded rather than a continuous fluorescent track limits the estimation of the number of repeats in the continuous tandem. Thus, in addition to the gaps observed, suggests several kilobases of unrelated sequence flanked by SSRs, the existence of several DNA kilobases of unrelated sequences within the fluorescence string of signals cannot be ruled out (Figure 5f).

Clear differences were observed after Southern blot hybridization. The pentanucleotides, found exclusively in the heterochromatin by ND-FISH, hybridized at the exclusion limit of the gel, indicating the existence of large arrays of these repeats in the enzyme-resistant DNA fraction (Figure 6B-E). In addition, the specific pattern of multiple fragments observed over a wide range of molecular weight probably reflects heterogeneity within the tandem repeats and/or the existence of other sequences present within these highly repetitive blocks. This agrees with the analysis of sequences of individual pentanucleotide SSR tracts, demonstrating that they could be imperfect and might be flanked by DNA sequences, mainly related to transposable elements $[8,33]$.

None of the SSRs with a repeat motif of less than four bases analysed by Southern blotting hybridized with the undigested DNA fraction, and no fragments with a molecular weight of over $10 \mathrm{~kb}$ were found (Figure 6F-K). This suggests that these SSRs must be organised in clusters of short tandem repeat stretches instead of the longer blocks that characterize the classic satellites. However, they provide the extended regions of several kilobases required to produce consistent in situ signals on mitotic chromosomes. The differences in genomic organisation suggested by the Southern blot analysis might explain why the novel SSRs clustered in the heterochromatin of $D$. melanogaster, have never been previously isolated or cloned using the satellite DNA fragments obtained by ultracentrifugation in caesium density gradients [8].

\section{Abundance and distribution of SSRs in D. melanogaster chromosomes}

The analysis of complete euchromatin sequences revealed that, as for most of the genomes examined to date, the $D$. melanogaster genome shows great variation in terms of the abundance and distribution of SSRs [34-37]. The present results for the polytene chromosomes agree with some of the above database-held information. For example, in the euchromatic chromosome arms, the SSRs based on the dinucleotides AC and AG were the most common, followed by the SSR with the mononucleotide A. In general, trinucleotide repeats were only detected in small amounts by ND-FISH (Figure 3a-k). These results might be explained by the size of the SSR loci and the abundance of the different motifs found in D. melanogaster genome. The frequency of the complete trinucleotide SSR set was found to be about half that of the AC repeat loci. Moreover, about $10 \%$ of the AC repeat loci have more than $100 \mathrm{bp}$ while trinucleotide SSRs loci with more than 50 bp are very rare and only the $\mathrm{ACT}$ and $\mathrm{AAT}$ repeats have more than 100 bp [38]. Therefore, a minimum target sequence size (long SSRs or high density of short loci) is required for ND-FISH signals to be detected even when using polytene chromosomes. As expected, because long pentanucleotide SSR loci are rare in D. melanogaster sequenced genome they were mainly localized in the chromocentre of polytene chromosomes (Figure 1). However, half of the SSRs scanned in this work, some of which were abundant in the heterochromatin, also had a significant presence in the euchromatin (Figure 2 and 3).

The present results show an uneven chromosome distribution for several motifs within and between the individual chromosomes of $D$. melanogaster. These motifs show a heterogeneous distribution with marked differences in density, both in the euchromatin and heterochromatin. This confirms earlier observations made by in situ hybridization showing a high density of SSRs, especially for the mono- and dinucleotide repeats in the euchromatic arm of chromosome $\mathrm{X}$ and their absence in chromosome 4, to correlate with the phenomena of gene dosage compensation and recombination $[39,40]$. The results of the present work suggest that both the absolute and relative frequencies of SSRs recorded to date in the $D$. melanogaster database would be significantly different if the whole-genome sequence were available. The high quantity of GATA repeats in the heterochromatin with respect to the euchromatic regions supports this assumption (Figure 3o-q). Moreover, with the exception of GATA repeats, the remaining SSRs were less abundant in the $\mathrm{X}$ than in the $\mathrm{Y}$ chromosome. The differences between sexes are notable with respect to some repeats such as AAGAG or 
AAGAC, which are particularly enriched in the Y chromosome (compare Figure $1 \mathrm{~b}$ and 1e). Previous molecular characterization of SSRs using total genomic DNA from embryos of $D$. melanogaster indicated that AAGAG and AATAC comprise 5.6\%, and $0.52 \%$ respectively of the genome [8]. The present ND-FISH results indicate that some SSRs motifs are very abundant in the heterochromatin of D. melanogaster, with AAGAG and AATAC the most and least common respectively.

\section{Conclusions}

Our ND-FISH method makes highly efficient the detection of SSRs in D. melanogaster chromosomes. The data clearly show the variation in the abundance of different SSR motifs and reveal their non-random distribution within and between chromosomes. As judged by our results, the greater representation of certain SSRs in D. melanogaster heterochromatin suggests that its complexity may be greater than previously thought. Thus, the possibility that these sequences may be implicated in some putative heterochromatin role(s) cannot be excluded.

\section{Acknowledgements}

This study was supported by grants from the Spanish Ministry of Science and Innovation (AGL 2009-10373). The authors thank Adrian Burton for linguistic assistance.

\section{Authors' contributions}

AC performed experiments, design the study and analysed data. AC and NJ wrote the paper. All authors read and approved the final manuscript.

Received: 3 February 2011 Accepted: 26 April 2011

Published: 26 April 2011

\section{References}

1. Grewal SI, Elgin SC: Heterochromatin: new possibilities for the inherence of structure. Curr Opin Genet Dev 2002, 12:178-187.

2. Morris CA, Moazed D: Centromere assembly and propagation. Cell 2007, 128(4):647-650.

3. Martienssen RA: Maintenance of heterochromatin by RNA interference of tandem repeats. Nat Genet 2007, 35(3):213-214.

4. Bosco G, Campbell P, Leiva-Neto JT, Markow TA: Analysis of Drosophila species genome size and satellite DNA content reveals significant differences among strains as well as between species. Genetics 2007, 177:1277-1290.

5. Plohl M, Luchetti A, Mestrovic N, Mantovani B: Satellite DNAs between selfishness and functionality: Structure, genomics and evolution of tandem repeats in centromeric (hetero)chromatin. Gene 2008, 409:72-82.

6. Hoskins, et al: Heterochromatic sequences in a Drosophila wholegenome shotgun assembly. Genome Biology 2002, 3:Research0085.1-16.

7. Zhang P, Spradling AC: The drosophila salivary gland chromocenter contains highly polytenized subdomains of mitotic heterochromatin. Genetics 1995, 2:659-670.

8. Lohe AR, Brutlag DL: Multiplicity of satellite DNA sequence in Drosophila melanogaster. Proc Natl Acad Sci USA 1986, 83:696-700.

9. Abad JP, Carmena M, Baars S, Saunders RDC, Glover DM, Ludeña P, Sentis C, Tyler-Smith C, Villasante A: Dodeca satellite: A conserved G+C rich satellite from the centromeric heterochromatin of Drosoohila melanogaster. Proc Natl Acad Sci USA 1992, 89:4663-4667.

10. Lohe AR, Hilliker AJ, Roberts PA: Mapping simple repeated DNA sequences in heterochromatin of Drosophila melanogaster. Genetics 1993, 134:1149-1174.
11. Makunin IV, Pokholkoa GV, Kholodilow NG, Zakharkin SO, Bonaccorsi S, Dimitri P, Zhimulev IF: A novel simple satellite DNA is colocalized with the Stalker retrotransposon in Drosophila melanogaster heterochromatin. Mol Gen Genen 1999, 261:381-387.

12. Celniker SE, et al: Finishing a whole-genome shotgun: Release 3 of the Drosophila melanogaster euchromatic genome sequence. Genome Biol 2002, 3:Research00791-14.

13. Hoskins RA, et al: Sequence finishing and mapping of Drosophila melanogaster heterochromatin. Science 2007, 16:1625-1628.

14. Tautz D, Renz M: Simple sequences are ubiquitous repetitive components of eukaryotic genomes. Nucleic Acids Res 1984, 4127-4138.

15. Jeffreys AJ, Wilson $V$, Thein SL: Hypervariable 'minisatelite' regions in human DNA. Nature 1985, 314:67-73.

16. Schäfer R, Ali S, Epplen JT: The organization of the evolutionarily conserved GATA/GACA repeats in the mouse genome. Chromosoma 1986, 93:502-510.

17. Cuadrado A, Schwarzacher T: The chromosomal organization of simple sequence repeats in wheat and rye genomes. Chromosoma 1999, 107:587-594

18. Epplen JT: On simple repeated GAT/CA sequences in animal genomes: A critical reappraisal. Journal of Heredity 1988, 79:409-417.

19. Guy J, Spallluto C, McMurray A, Hearn T, et al: Genomic sequence and transcriptional profile of the boundary between pericentromere satellites and genes on human chromosome arm 10p. H Mol Genet 2000, 9(13):2029-2042

20. Hancock JM: Genome size and the accumulation of simple sequence repeats: implications of new data from genome sequencing projects. Genetica 2002, 115:93-103.

21. Cuadrado $\mathrm{N}$, Jouve $\mathrm{N}$ : Chromosomal detection of simple sequence repeats (SSRs) using nondenaturing FISH (ND-FISH). Chromosoma 2010, 19:495-503.

22. Fidlerová $H$, Senger G, Kost M, Sanséau P, Sherr D: Two simple procedures for releasing chromatin from routinely fixed cells for fluorescence in situ hybrization. Cytogenet Cell Genet 1994, 65:203-2005.

23. Wiegant J, Kalle W, Mullenders L, Brookes S, Hoovers JMN, Dauwerse JG, van Ommen GJB, Raap AK: High-resolution in situ hybridization using DNA halo preparations. Hum Mol Genet 1992, 1:587-591.

24. Kuhn GCS, Teo CH, Schwarzacher T, Heslop-Harrison JS: Evolutionary dynamics and sites of illegitimate recombination revealed in the interspersion and sequence junctions of two nonomologous satellites DNAs in cactophilic Drosophila species. Heredity 2009, 102:453-464.

25. Loarce $Y$, Hueros G, Ferrer E: A molecular linkage map of rye. Theor Appl Genet 1996, 93:1112-1118.

26. Pimpinelli S, Berloco M, Fanti L, Dimitri P, Bonaccorsi S, Marchetti E, Caizzi R, Caggese C, Gatti M: Transposable elements are stable structural components of Drosophila melanogaster heterochromatin. Proc Natl Acad Sci USA 1995, 92:3804-3808.

27. Cuadrado A, Jouve N: Similarities in the chromosomal distribution of AG and $A C$ repeats within and between Drosophila, human and barley chromosomes. Cytogenet Genome Res 2007, 119:91-99.

28. Smith CD, Shy S, Mungall CJ, Karpen GH: The release 5.1 annotation of Drosophila melanogaster heterochromatin. Science 2007, 316:1586-1591.

29. Mardis e, McPherson J, Martienssen R, Wolson R, McCombie WR. What is finished, and why does it matter. Genome Research 2002, 12:669-671.

30. O'Hare K, Chadwick BP, Constantinou A, Davis Aj, Mitchelson A, Tudor M: A 5,9-kb tandem repeat at the euchromatin-heterochromatin boundary of the X chromosome of Drosophila melanogaster. Mol Genet Genomics 2002, 267:657-6.

31. Singh L, Phillips $C$, Jones KW: The conserved nucleotide sequences of Bkm, which define sxr in the mouse, are transcribed. Cell 1984, 35:111-120.

32. Le MH, Duricka D, Karpen GH: Islands of complex DNA are widespread in Drosophila centric heterochromatin. Genetics 1995, 141:283-303.

33. Sun X, Le HD, Wahlstrom JM, Karpen GR: Sequence analysis of a functional Drosophila centromere. Genome Research 2003, 13:82-194.

34. Bachtrog D, et al: Distribution of dinucleotide microsatellites in the Drosophila melanogaster genome. Mol Biol Evo 1999, 16:602-610.

35. Toth G, Gaspari Z, Jurka J: Microsatellites in different eukaryotic genomes: survey and analysis. Genome Res 2000, 10:967-981. 
36. Katti MV, Ranjekar PK, Gupta VS: Differential distribution of Simple Sequence Repeats in eukaryotic genome sequences. Mol Biol Evol 2001, 18:1161-1167.

37. Subramanian S, Mishra RK, Singh L: Genome-wide analysis of microsatellite repeats in humans: their abundance and density in specific genomic regions. Genome Biology 2003, 4:R13.

38. Subirana $J A$, Messenguer $X$ : The most frequent short sequences in noncoding DNA. Nucleic Acids Research 2010, 38:1172-1181.

39. Pardue ML, Lowenhaupt K, Rich A, Nordheim A: (dC-dA)n.(dG-dT)n sequences have evolutionarily conserved chromosomal locations in Drosophila with implications for roles in chromosome structure and function. EMBO J 1987, 6:1781-1789.

40. Lowenhaupt K, Rich A, Pardue ML: Nonrandom distribution of long mono- and dinucleotide repeats in Drosophila chromosomes: Correlations with dosage compensation, heterochromatin and recombination. Molecular and Cellular Biology 1989, 9:1173-1182.

doi:10.1186/1471-2164-12-205

Cite this article as: Cuadrado and Jouve: Novel simple sequence repeats (SSRs) detected by ND-FISH in heterochromatin of Drosophila melanogaster. BMC Genomics 2011 12:205.

\section{Submit your next manuscript to BioMed Central} and take full advantage of:

- Convenient online submission

- Thorough peer review

- No space constraints or color figure charges

- Immediate publication on acceptance

- Inclusion in PubMed, CAS, Scopus and Google Scholar

- Research which is freely available for redistribution

Submit your manuscript at www.biomedcentral.com/submit
C Biomed Central 